CASO CLÍNICO

\title{
Síndrome de Bannayan-Riley-Rubalcaba en pediatría
}

\author{
Beth Sainz de la Peña-Hernández ${ }^{1 *}$ y Bertha E. Guillén-Palacios ${ }^{2}$ \\ ${ }^{1}$ Departamento de Pediatría; ${ }^{2}$ Departamento de Dermatología Pediátrica. Hospital de Especialidades Pediátricas, Tuxtla Gutiérrez, Chiapas, México
}

\section{Resumen}

Introducción: El síndrome de Bannayan-Riley-Ruvalcaba (SBRR) forma parte de la enfermedad de PTEN tumor-hamartoma, que comprende los síndromes de Cowden, Proteus y similar a Proteus, los cuales presentan un espectro de lesiones cutáneas, mucosas, de mama, tiroides y tracto gastrointestinal, así como polipomatosis hereditaria autosómica dominante. El SBRR se caracteriza por macrocefalia, lipomatosis, hemangiomatosis, pólipos intestinales, lentiginosis genital y discapacidad intelectual. El diagnóstico clínico y de variantes patogénicas en el gen PTEN, detectables en el 60\% de los afectados, brinda la oportunidad de un manejo adecuado y de asesoramiento genético. Caso clínico: Se reporta el caso de un paciente en edad escolar que fue enviado a valoración inicial a dermatología por presentar antecedente de macrocefalia al nacimiento, lentiginosis genital, retraso en el desarrollo psicomotor y posteriormente rectorragia secundaria a polipomatosis intestinal. Se le realizó el diagnóstico clínico y molecular de SBRR. Conclusiones: El SBRR es poco frecuente, lo que puede retrasar el diagnóstico para los pacientes y los familiares en riesgo, por lo que es importante conocer sus características clínicas en el paciente pediátrico para lograr un diagnóstico y un manejo oportunos.

Palabras clave: Síndrome Bannayan-Riley-Ruvalcaba. PTEN. Polipomatosis. Lentiginosis genital. México.

\section{Bannayan-Riley-Rubalcaba syndrome in pediatrics}

\begin{abstract}
Background: Bannayan-Riley-Ruvalcaba syndrome (BRRS) is part of the PTEN tumor-hamartoma disease, which includes the Cowden, Proteus and Proteus-like syndromes, which present a spectrum of skin, mucosal, breast, thyroid, and gastrointestinal tract lesions, as well as autosomal dominant hereditary polypomatosis. BRRS is characterized by macrocephaly, lipomatosis, hemangiomatosis, intestinal polyps, genital lentiginosis, and intellectual disability. Clinical diagnosis and diagnosis of pathogenic variants in the PTEN gene, detectable in $60 \%$ of those affected, provides the opportunity for appropriate management and genetic counseling. Case report: We report the case of a school-age patient who was sent to an initial dermatological evaluation for presenting a history of macrocephaly at birth, genital lentiginosis, delayed psychomotor development and later rectal bleeding secondary to intestinal polypomatosis. A clinical and molecular diagnosis of BRRS was carried out. Conclusions: BRRS is rare, which can delay the diagnosis for patients and relatives at risk, so it is important to know its clinical characteristics in pediatric patients to achieve a timely diagnosis and management.
\end{abstract}

Key words: Bannayan-Riley-Ruvalcaba syndrome. PTEN. Hamartomatous polyps. Genital lentiginosis. Mexico.

\section{Correspondencia:}

*Bertha E. Guillén-Palacios

E-mail: guillenpa@ hotmail.com
Fecha de recepción: 20-06-2020

Fecha de aceptación: 06-11-2020

DOI: 10.24875/BMHIM.20000172
Disponible en internet: 27-04-2021 Bol Med Hosp Infant Mex. 2021;78(3):245-250

www.bmhim.com 1665-1146/@ 2020 Hospital Infantil de México Federico Gómez. Publicado por Permanyer. Este es un artículo open access bajo la licencia CC BY-NC-ND (http://creativecommons.org/licenses/by-nc-nd/4.0/). 


\section{Introducción}

En 1960, Riley y Smith ${ }^{1}$ agruparon la tríada de macrocefalia, pseudopapiledema y hemangiomas. En 1971, Bannayan ${ }^{2}$ describió el conjunto de macrocefalia, lipomatosis y hemangiomas, mientras que Ruvalcaba, en 1980, asoció macrocefalia, pólipos hamartomatosos intestinales, lentiginosis genital y discapacidad intelectual. Finalmente, en 1990, Cohen ${ }^{4}$ sugirió que todas estas características podrían entrar en una única enfermedad denominada síndrome de Bannayan-RileyRuvalcaba (SBRR; OMIM158350). El SBRR se incluye entre los síndromes de PTEN y tumores hamartomatosos, en el cual se agrupan otros como los síndromes de Cowden y de Proteus ${ }^{1-4}$.

En este reporte se presenta un caso de SBRR diagnosticado en un hospital de tercer nivel de atención de México, al mismo tiempo que se realiza una breve revisión de la literatura para familiarizar a los médicos generales y a los pediatras con esta enfermedad de baja prevalencia con predisposición a diversas neoplasias.

\section{Caso clínico}

Paciente de sexo masculino, producto de primera y única gesta, con ambos progenitores aparentemente sanos, no consanguíneos; se desconocen más datos del padre biológico. Control prenatal referido como adecuado; la madre cursó con infección de vía urinarias en el primer trimestre, y el resto del embarazo se reportó sin complicaciones. A falta de progresión del trabajo de parto, se realizó cesárea secundaria sin complicaciones al nacimiento. La somatometría neonatal indicó un peso de $3800 \mathrm{~g}$. Al nacimiento se detectó macrocefalia, pero la madre no recuerda el perímetro cefálico. El binomio egresó sin eventualidades. Los hitos del desarrollo se refirieron con la presencia de bisílabos a los 9 meses, bipedestación a los 15 meses y control de esfínteres a los 4 años.

El paciente acudió por primera vez al Hospital de Especialidades Pediátricas a los 4 años de edad por presentar hipertrofia amigdalina de grado IV con amigdalitis crónica, por lo que fue programado por el servicio de otorrinolaringología para realización de adenoamigdalectomía en julio de 2012. A los 6 años, se detectó la presencia de máculas pigmentadas de color café en los genitales, por lo fue atendido por un médico en el Centro de Salud de Bochil, quien lo refirió al servicio de dermatología del Hospital de Especialidades Pediátricas, en Tuxtla.

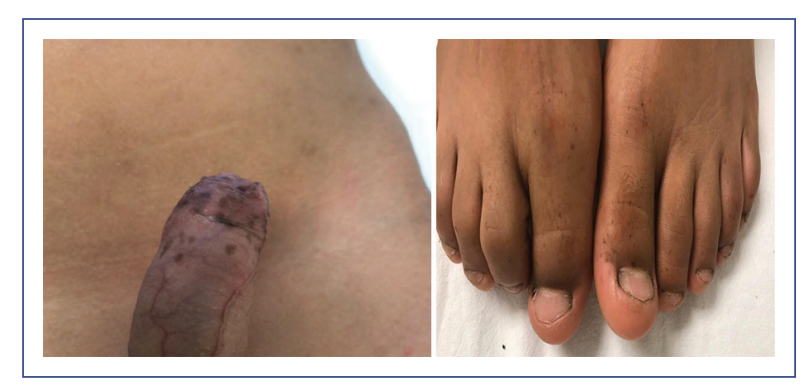

Figura 1. A: lentiginosis en zona genital. B: lentiginosis en ortejos.

En la exploración física se detectó macrocefalia con un perímetro cefálico de $57 \mathrm{~cm}$ (> P75), peso de $16.6 \mathrm{~kg}$, talla de $100 \mathrm{~cm}$, pequeñas máculas de color café diseminadas en la cara, el tronco y las extremidades, que afectaban los labios, el pene (Figura 1 A) y los ortejos (Figura $1 \mathrm{~B}$ ), la mayoría entre 2 y $4 \mathrm{~mm}$, y presencia de extremidades con braquidactilia. En ese momento se consideraron diagnósticos diferenciales de síndrome de Peutz-Jeghers y SBRR. Se exploró de forma dirigida a la madre, sin encontrar estigmas cutáneos. Durante el seguimiento, el paciente presentó rectorragia recurrente, por lo que se le practicó una colonoscopia. Se resecaron dos pólipos rectales, con reporte de anatomía patológica como pólipos rectales juveniles, el mayor de $0.7 \times 0.5 \mathrm{~cm}$ y el menor de 0.5 $\times 0.4 \mathrm{~cm}$, sin evidencia de neoplasia. Continuando con el abordaje diagnóstico, se realizó de forma externa un análisis molecular en sangre periférica mediante exoma dirigido para síndromes de predisposición a cáncer hereditario de 94 genes, basado en la tecnología de captura con sondas de oligonucleótidos (Nextera Rapid Capture Illumina Inc.) y secuenciación de siguiente generación en el equipo MiSeq. Se encontró la variante NM_000314.6:c.955delA del gen PTEN (phosphatase and tensin homolog deleted on chromosome 10), que origina el cambio en la proteína NP_000305.3:P. Thr319LefusTer2. Para el análisis bioinformático se emplearon los programas Trimmomatic, BWA (algoritmo mem) y GATK, y para la anotación de las variantes se empleó SnpEff, Mutation Taster e información de bases de datos y de la literatura internacional. La variante se clasificó como patogénica, pese a que no ha sido reportada en dbSNP, ClinVar del NCBI (National Center for Biotechnology Information) ni HGMD (Human Gene Mutation Database) (último acceso en agosto del 2020). Sin embargo, otras variantes que afectan al nucleótido 955 por deleción o duplicación han sido reportadas y clasificadas como patogénicas. No se 
Tabla 1. Cronología de los hallazgos y valoraciones del caso

\begin{tabular}{|c|c|c|}
\hline Aparatos y sistemas & Edad de presentación & Hallazgos \\
\hline Neurológico & $\begin{array}{l}\text { 1. Al nacimiento } \\
\text { 2. Detectado en febrero de } \\
2017 \text { por psicología } \\
\text { 3. Detectado en febrero de } \\
2017 \text { por psicología }\end{array}$ & $\begin{array}{l}\text { 1. Macrocefalia } \\
\text { 2. Déficit de atención de acuerdo con el Kiddie Schedule for Affective Disorders } \\
\text { and Schizophrenia } \\
\text { 3. Capacidad intelectual limítrofe con coeficiente intelectual de } 74 \text { según la } \\
\text { Escala de Wechsler de inteligencia para niños }\end{array}$ \\
\hline Cardiovascular & Sin problemas detectados & Sin problemas detectados \\
\hline Respiratorio & 4 años & $\begin{array}{l}\text { Infecciones de vías respiratorias altas de repetición, se detecta hipertrofia } \\
\text { amigdalina de grado IV con amigdalitis crónica (amigdalectomía) }\end{array}$ \\
\hline Gastrointestinal & 10 años & $\begin{array}{l}\text { Rectorragia } \\
\text { Colonoscopia: poliposis múltiple + polipectomía + cauterización de pólipos } \\
\text { sésiles. Se observa en recto y sigmoides múltiples pólipos sésiles y un par } \\
\text { pediculados de } 5 \mathrm{~mm}\end{array}$ \\
\hline Endocrinología & Sin problemas detectados & Sin problemas detectados \\
\hline Tegumentario & 6 años & $\begin{array}{l}\text { Máculas pigmentadas de color café en cara, tronco y extremidades que afectan } \\
\text { labios, pene, mucosa genital y ortejos }\end{array}$ \\
\hline Renal & Sin problemas detectados & Sin problemas detectados \\
\hline
\end{tabular}

realizó ningún análisis in silico, ya que no se cuenta con el recurso. A la madre del paciente se le realizó el estudio molecular en búsqueda de la variante encontrada; sin embargo, se reportó como normal y se brindó asesoramiento genético.

De acuerdo con los hallazgos clínicos y moleculares se realizó el diagnóstico de SBRR de novo.

En la Tabla 1 se resumen las valoraciones y los abordajes por aparatos y sistemas, con su cronología.

\section{Discusión}

El SBRR es una enfermedad poco frecuente; en todo el mundo se han reportado cerca de 100 casos, con una incidencia de 1 en 200,000. Sin embargo, la cifra podría ser mayor debido a la dificultad para realizar el diagnóstico ante síntomas inespecíficos ${ }^{5-7}$. Se ha observado un predominio de casos en el sexo masculino que podría relacionarse con las máculas hiperpigmentadas en el pene como hallazgo específico ${ }^{8}$.

El SBRR se origina por variantes patogénicas en línea germinal del gen PTEN, que en su mayoría corresponden a un patrón de herencia autosómico dominante; sin embargo, el $10-45 \%$ de los casos son de novo ${ }^{9}$. El $65 \%$ de las variantes patogénicas se encuentran en regiones codificantes y el $11 \%$ se deben a duplicaciones o deleciones que, en su mayoría, recaen en el exón 5 , en el centro catalítico de la fosfatasa. Recientemente se han reportado en el exón 9, ya que no se habían documentado variantes ${ }^{10}$. En diversos tumores (tiroides, mama, endometrio, próstata y riñón) se han encontrado mutaciones somáticas en el gen PTEN ${ }^{10}$.

El gen PTEN, locus 10q23, consta de nueve exones y se expresa de forma ubicua en diversos órganos, en particular en el tejido graso y el bazo. PTEN codifica una fosfatasa con sustratos lipídicos y proteicos con actividad constitutiva de supresor tumoral ${ }^{7}$, facilitando la apoptosis o el arresto del ciclo celular en la fase G1 mediante la actividad fosfatasa, y regulando de forma negativa las concentraciones intracelulares de fosfatidilinositol trifosfato, así como la vía de señalización de $\mathrm{AKT} / \mathrm{PKB}^{11}$. Las mutaciones de este gen resultan en síndromes de sobrecrecimiento y malignidad, por lo cual se ha sugerido mantener el tamizaje ante el riesgo aumentado de desarrollar cáncer de mama, tiroides, endometrio, carcinomas renales y melanoma cutáneo. Se han observado mutaciones de PTEN en numerosas neoplasias esporádicas (hematológicas y sólidas) ${ }^{11,12}$. Clínicamente, se presentan con múltiples lipomas, hemangiomas, polipomatosis intestinal hamartomatosa, malformaciones arteriovenosas, retraso en el neurodesarrollo, autismo, macrocefalia y lentiginosis genital. Otras características que se han asociado son hueso frontal prominente, hipertelorismo, alteraciones tiroideas, alteraciones retinianas, escoliosis, pectus excavatum, hiperlaxitud articular y miopatía proximal con hipotonía ${ }^{13,14}$.

Aunque no existen criterios diagnósticos definidos, Parisi, et al..$^{14}$ propusieron al menos dos de los 
Tabla 2. Criterios diagnósticos para síndromes de PTEN y tumores hamartomatosos ${ }^{16}$

\section{Criterios diagnósticos para PHTS}

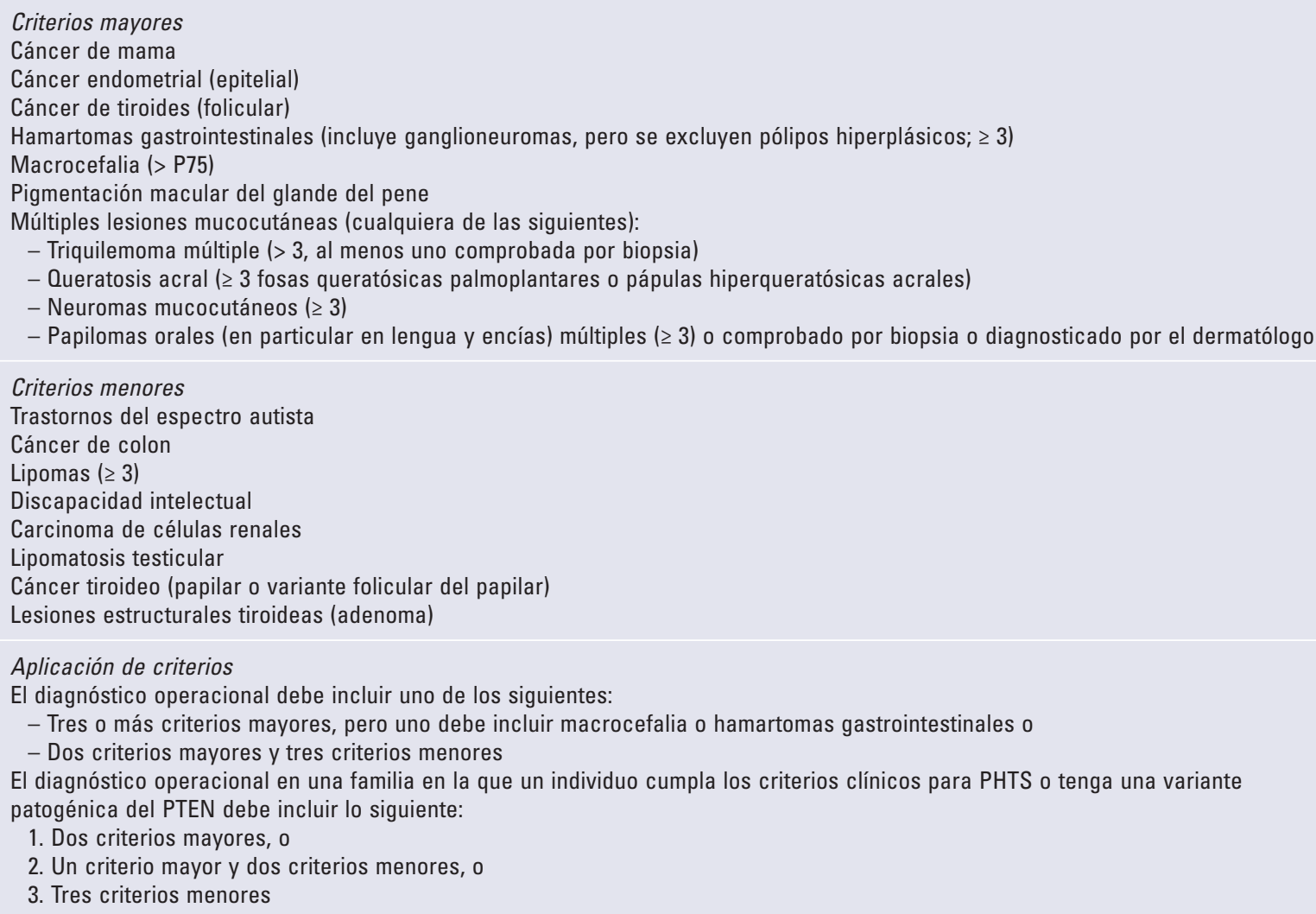

PHTS: síndrome de tumor hamartoma PTEN.

siguientes: macrocefalia, hamartomas (incluyendo al menos un lipoma, hemangioma o pólipo intestinal) y máculas en el pene para pacientes de sexo masculino. El diagnóstico se confirma mediante la identificación de mutaciones en el gen PTEN ${ }^{15}$. Pilarski, et al. ${ }^{16}$ han propuesto algunos criterios diagnósticos para síndromes de PTEN y tumores hamartomatosos (Tabla 2).

Se han demostrado mutaciones idénticas en PTEN en pacientes con síndrome de Cowden. Existen familias con superposición de síndrome de Cowden y SBRR, con miembros que manifiestan ambos fenotipos. Debido a tal superposición clínica y genética, el SBBR y el síndrome de Cowden ahora se aceptan como expresiones fenotípicas diferentes del mismo síndrome alélico y se denominan colectivamente síndrome de hamartoma-tumor PTEN ${ }^{16}$. Se han reportado todos los tipos de variantes patogénicas en las mutaciones de PTEN (deleciones completas del exón, pérdida de función, sentido equivocado y promotor), aunque sin una clara correlación genotipo-fenotipo, ya que incluso pacientes con la misma variante patogénica presentan fenotipos distintos del $\mathrm{SBRR}^{16}$. Ante la falta de una correlación firme genotipo-fenotipo, es recomendable que los pacientes con una variante de PTEN patógena o probablemente patógena presente en la infancia sigan las pautas de vigilancia para la detección oportuna de cáncer a medida que envejecen ${ }^{16-18}$.

En este reporte se expone el caso de un paciente que acudió por primera vez a la consulta de dermatología por lentiginosis genital, asintomático, en quien se sospechó el diagnóstico de SBRR por las máculas en el pene y la macrocefalia. Posteriormente, en el abordaje integral se detectaron problemas conductuales, retraso del desarrollo y discapacidad intelectual. Además, en un periodo corto, el paciente presentó rectorragia secundaria y polipomatosis intestinal. Se detectó una variación de la mutación del gen PTEN que incluye un número importante de las alteraciones descritas para este síndrome.

En México se tienen reportados muy pocos casos de SBRR. En una revisión de reportes de caso se identificaron al menos cinco, de los cuales cuatro se 


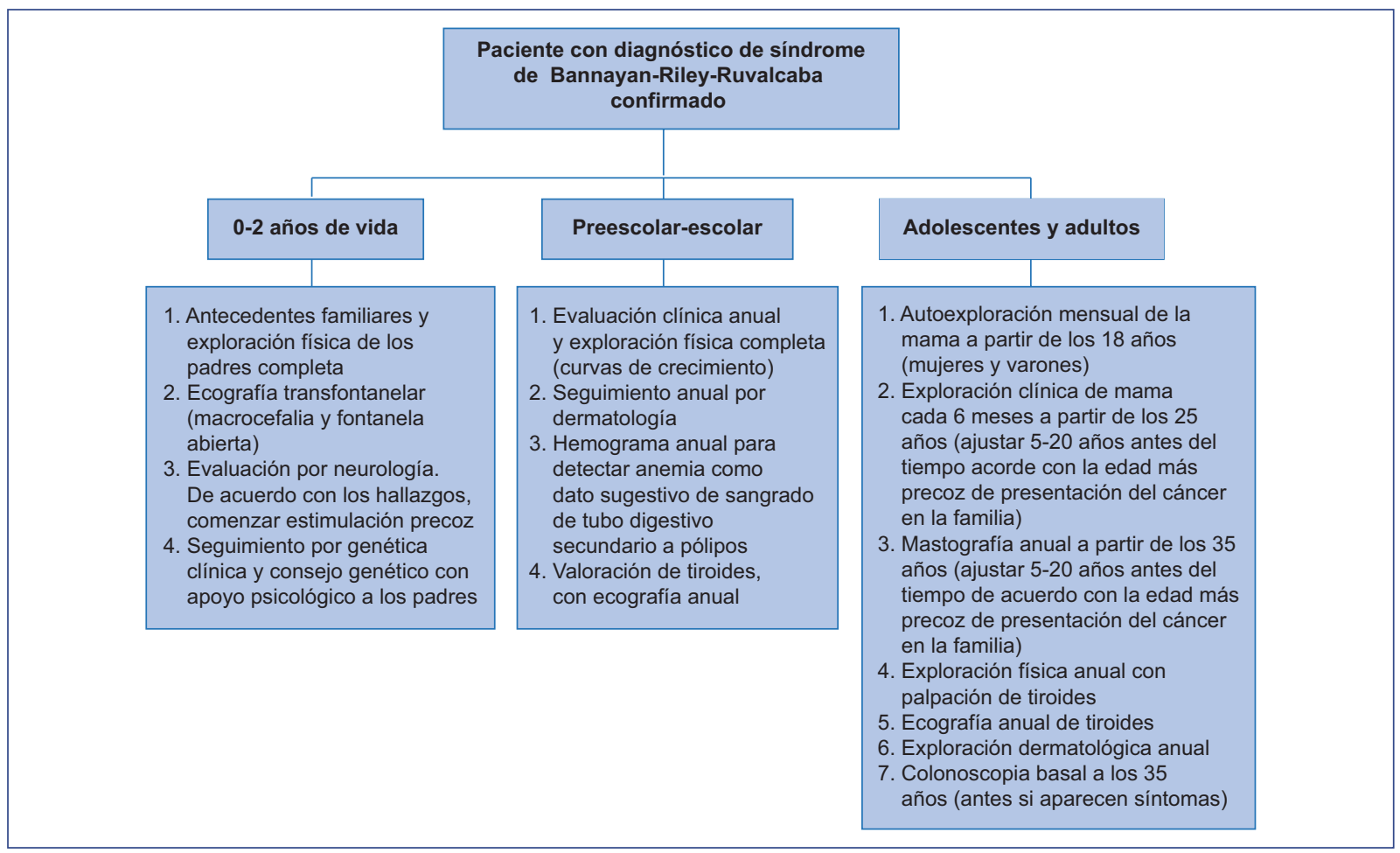

Figura 2. Diagrama de flujo para el seguimiento del paciente con síndrome de Bannayan-Riley-Rubalcaba.

reportaron en un estudio de polipomatosis gastrointestinal en pediatría. El otro caso correspondió a un estudio de manchas de color café con leche en una consulta externa de dermatología ${ }^{19,20}$.

Familiarizarse con este tipo de enfermedades poco frecuentes permitirá buscar de manera intencionada el diagnóstico del paciente: los hallazgos clínicos descritos (macrocefalia, lentiginosis genital, retardo del neurodesarrollo y discapacidad intelectual) por sí solos llevarán a la sospecha de este síndrome, el cual debe ser confirmado con estudios moleculares. En la Figura 2 se propone un flujograma para el seguimiento de los pacientes con SBRR de acuerdo con la edad.

La importancia del diagnóstico de SBRR recae en su asociación con el desarrollo de neoplasias en la edad adulta y con otras complicaciones que requieren cribado para su detección y manejo oportuno.

\section{Responsabilidades éticas}

Protección de personas y animales. Los autores declaran que para esta investigación no se han realizado experimentos en seres humanos ni en animales.

Confidencialidad de los datos. Los autores declaran que han seguido los protocolos de su centro de trabajo sobre la publicación de datos de pacientes.
Derecho a la privacidad y consentimiento informado. Los autores han obtenido el consentimiento informado de los pacientes o sujetos referidos en el artículo. Este documento obra en poder del autor de correspondencia.

\section{Financiamiento}

Ninguno.

\section{Conflicto de intereses}

Los autores declaran no tener ningún conflicto de intereses.

\section{Bibliografía}

1. Riley HD, Smith WR. Macrocephaly, pseudopapilledema and multiple hemangiomata. Pediatrics. 1960;26:293-300.

2. Bannayan GA. Lipomatosis, angiomatosis, and macrencephalia. A previously undescribed congenital syndrome. Arch Pathol. 1971;92:1-5.

3. Zigman AF, Lavine JE, Jones MC, Boland CR, Carethers JM. Localization of the Bannayan-Riley-Ruvalcaba syndrome gene to chromosome 10q23. Gastroenterology. 1997;113:1433-7.

4. Cohen MM. Bannayan-Riley-Ruvalcaba syndrome: renaming three formerly recognized syndromes as one etiologic entity. Am J Med Genet. 1990;35:291.

5. Hendriks YMC, Verhallen JTCM, van der Smagt JJ, Kant SG, Hilhorst Y, Hoefsloot L, et al. Bannayan-Riley-Ruvalcaba syndrome: further delineation of the phenotype and management of PTEN mutation-positive cases. Fam Cancer. 2003;2:79-85. 
6. Lachlan KL, Lucassen AM, Bunyan D, Temple IK. Cowden syndrome and Bannayan Riley Ruvalcaba syndrome represent one condition with variable expression and age-related penetrance: results of a clinical study of PTEN mutation carriers. J Med Genet. 2007:44:579-85.

7. Schreibman IR, Baker M, Amos C, McGarrity TJ. The hamartomatous polyposis syndromes: a clinical and molecular review. Am J Gastroenterol. 2005;100:476-90.

8. Blumenthal GM, Dennis PA. PTEN hamartoma tumor syndromes. Eur J Hum Genet. 2008;16:1289-300.

9. Nosé V. Genodermatosis affecting the skin and mucosa of the head and neck: clinicopathologic, genetic, and molecular aspect PTEN-Hamartoma Tumor Syndrome/Cowden Syndrome. Head Neck Pathol. 2016; 10:131-8.

10. Pezzolesi MG, Li Y, Zhou XP, Pilarski R, Shen L, Eng C. Mutation-positive and mutation-negative patients with Cowden and Bannayan-Riley-Ruvalcaba syndromes associated with distinct $10 q$ haplotypes. Am J Hum Genet. 2006;79:923-34.

11. Zhou XP, Waite KA, Pilarski R, Hampel H, Fernandez MJ, Bos $\mathrm{C}$, et al Germline PTEN promoter mutations and deletions in Cowden/Bannayan-Riley-Ruvalcaba syndrome result in aberrant PTEN protein and dysregulation of the phosphoinositol-3-kinase/Akt pathway. Am J Hum Genet. 2003;73:404-11.

12. Tan MH, Mester JL, Ngeow J, Rybicki LA, Orloff MS, Eng C. Lifetime cancer risks in individuals with germline PTEN mutations. Clin Cancer Res. 2012;18:400-7.
13. Hobert JA, Eng C. PTEN hamartoma tumor syndrome: an overview. Genet Med. 2009;11:687-94.

14. Parisi MA, Dinulos MB, Leppig KA, Sybert VP, Eng C, Hudgins L. The spectrum and evolution of phenotypic findings in PTEN mutation positive cases of Bannayan-Riley-Ruvalcaba syndrome. J Med Genet. 2001;38:52-8.

15. Marsh DJ, Kum JB, Lunetta KL, Bennett MJ, Gorlin RJ, Ahmed SF, et al. PTEN mutation spectrum and genotype-phenotype correlations in Bannayan-Riley-Ruvalcaba syndrome suggest a single entity with Cowden syndrome. Hum Mol Genet. 1999;8:1461-72.

16. Pilarski R, Burt R, Kohlman W, Pho L, Shannon KM, Swisher E. Cowden syndrome and the PTEN hamartoma tumor syndrome: systematic review and revised diagnostic criteria. J Natl Cancer Inst. 2013;105:1607-16.

17. Wanner M, Celebi JT, Peacocke M. Identification of a PTEN mutation in a family with Cowden syndrome and Bannayan-Zonana syndrome. J Am Acad Dermatol. 2001;44:183-7.

18. Macken WL, Tischkowitz M, Lachlan KL. PTEN hamartoma tumor syndrome in childhood: a review of the clinical literature. Am J Med Genet. 2019;181C:591-610.

19. Cázeres-Méndez JM, Zamudio-Vázquez VP, Gómez-Morales E, Ortiz-Aguirre SG, Cadena-León JF, Toro-Monjaraz EM. Pólipos gastrointestinales en pediatría. Acta Pediatr Mex. 2015;36:158-63.

20. Pérez-Hernández J, Frías-Ancona G, Vergara-López A. Prevalencia de las manchas café con leche en pacientes de consulta externa del servicio de dermatología del Centro Médico Nacional 20 de Noviembre. Dermatol Rev Mex. 2010;54:315-20. 